\title{
Fulminant Emphysematous Pyelonephritis: Case Report and Revision of the
}

\section{Literature}

\section{Pepe $P^{*}$, Pepe L, Panella P, Candiano G, Savoca F and Pennisi M}

Urology Unit, Cannizzaro Hospital, Catania, Italy

${ }^{*}$ Corresponding author: Pepe P, Urology Unit, Cannizzaro Hospital, Via Messina 829, Catania, Italy, Fax: +3995 7263259, Tel: +39 957263285, E-mail: piepepe@hotmail.com

Citation: Pepe P, Pepe L, Panella P, Candiano G, Savoca F, et al. (2021) Fulminant Emphysematous Pyelonephritis: Case Report and Revision of the Literature. J Case Rep Stud 9(2): 203

Received Date: November 17, 2021Accepted Date: December 08, 2021 Published Date: December 10, 2021

\begin{abstract}
A 56-year-old woman with type 2 diabetes mellitus and multiple sclerosis presented to our emergency department with right flank pain, fever $\left(38.6^{\circ}\right)$ and dysuria. A computed tomography (CT) scan showed the right kidney hydronephrosis secondary to ureteral stone, therefore a percutaneous nephrostomy was placed. The next day the patient's condition deteriorated rapidly; a new CT showed the presence of gas in the right kidney suspicious for emphysematous pyelonephritis (EPB) that needed an emergency nephrectomy. Thirtyfive days later the patient was discharged in good general condition.

In patients with urinary sepsis and worsening clinical conditions an early CT evaluation should be performed to exclude the presence of EPB.
\end{abstract}

Keywords: Emphysematous Pyelonephritis; Nephrectomy; Fulminant Pyelonephritis 


\section{Introduction}

Emphysematous pyelonephritis (EPN) is an acute necrotizing parenchymal secondary to gas-forming uropathogens and mortality rates in medically managed patients are as high as 70-90\% [1]; it should be suspected in diabetic and in immunosuppressed patients with urinary tract infections and worsening of clinical conditions. Rarely, a urinary tract infection refractory to medical treatment might warrant urgent surgical nephrectomy secondary to sepsis.

We report a 56-year-old woman who presented with urinary sepsis that needed emergency nephrectomy in view of her worsening condition.

\section{Case presentation}

A 56-year-old woman, a known type 2 diabetes mellitus in treatment with oral hypoglycemics, multiple sclerosis in therapy with immunosuppressive drugs presented to our emergency department with right flank pain for 8 days, associated with fever $\left(38.6^{\circ}\right)$ and dysuria. At admission, she was disoriented; blood exams showed sugar levels of $238 \mathrm{mg} \%$, urea $85 \mathrm{mg} \%$, serum creatinine $1.3 \mathrm{mg} \%$, total leukocyte count $15,200 / \mathrm{mm}^{3}$, and a platelet count of $116,000 / \mathrm{mm} 3$, C-reactive protein (PCR 9.3) and procalcitonine 0.4 . Urine examination was positive for sugar and ketones, and it showed proteinuria. An ultrasound of the abdomen showed hydronephrosis of the right kidney; a computed tomography (CT) scan of the abdomen performed without contrast medium showed hydronephrosis of the right kidney (Figure 1a) secondary to a stone placed in the homolateral iuxtavesical ureter. The women underwent antibiotic therapy and a percutaneous nephrostomy was placed in the right kidney. The next day, however, the patient's condition started to deteriorate rapidly; she was febrile $\left(39.2^{\circ}\right)$, drowsy, disoriented, with tachycardia, anuria, blood pressure of $80 / 55 \mathrm{mmHg}$ and tachypnea. She also had right flank pain, serum creatinine $4.3 \mathrm{mg} \%$, total leukocyte equal to $28,200 / \mathrm{mm} 3$, and a platelet count of $23,000 /$ $\mathrm{mm} 3$, PCR was 36 and procalcitonine 52, International Normalized Ratio (INR) 2.8. The women underwent CT scan that showed parenchymal destruction in the right kidney with presence of streaky or mottled gas radiating from the medulla to the cortex, characteristic of emphysematous pyelonephritis (Figure $1 \mathrm{~b}, \mathrm{c}$ and $\mathrm{d}$ ). The patient was submitted to an emergency right nephrectomy and was recovered in intensive care unit for vasopressor support, strict fluid balance, continuous veno-venous haemodialysis and insulin infusion resuscitation. A multidrug intravenous antibiotic therapy (meropemem, metronidazole and aminoglycoside) pending urine culture and blood culture was started; following urine culture positive for Klebsiella Pneumoniae a targeted antibiotic therapy was administrated. During the recovery the women underwent six blood bags transfusion secondary a voluminous hematoma located in the right retroperitoneum. The patient was initially recovered for 25 days in the intensive care unit and after a total of 35 days was discharged from our Department with good clinical parameters. Definitive specimen showed the presence of an acute and extensive pyelonephritis.

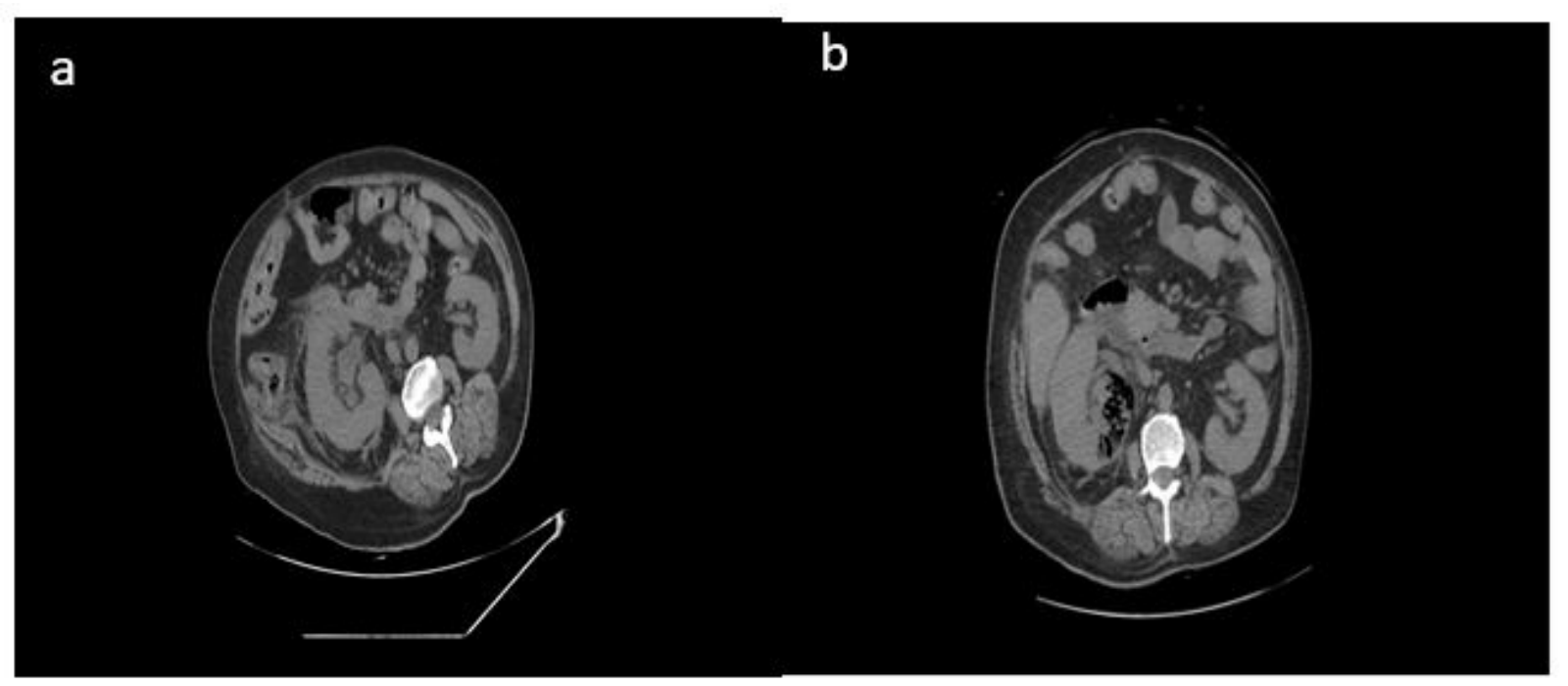



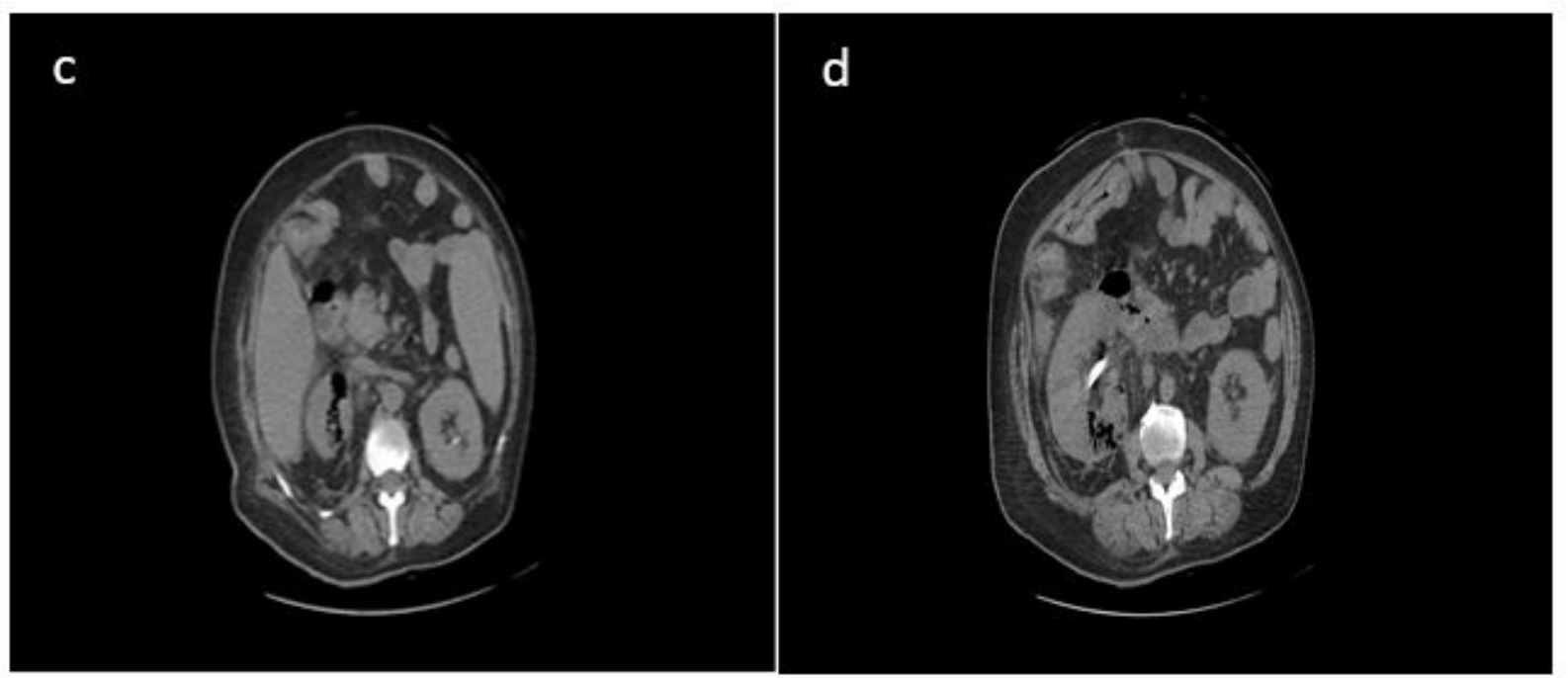

Figure 1: Axial sections of the CT scan showing absence (a) and presence of gas (24 hours later) following emphysematous pyelonephritis (b); presence of gas in the right kidney (c) after percutaneous nephrostomy placement (d)

\section{Discussion}

The most prevalent causative organism of EPN is Escherichia coli (69\% of the cases) followed by Klebsiella pneumonia (29\%) of the cases; Escherichia Coli metabolizes lactose and glucose to produce high levels of carbon dioxide and hydrogen resulting in significant gas formation [2]. Uncontrolled diabetes mellitus and ureteric obstruction predispose to severe infection [3]; in addition, defective immune response may worsen the clinical prognosis. The usual clinical presentation of EPN is acute and severe; although sometimes, a chronic infection could precede the acute attack. Urine cultures are invariably positive, but the diagnosis is established radiographically [4]. On abdominal radiographs, tissue gas in the renal parenchyma may appear as mottled gas shadows over the involved kidney; as the infection progresses, gas extends to the perinephric space and retroperitoneum. This distribution of gas should not be confused with "emphysematous pyelitis" in which air is in the collecting system of the kidney secondary to a gas-forming bacterial urinary tract infection; this infection often responds to antimicrobial therapy resulting in a better prognosis. Ultrasonography usually demonstrates strong focal echoes suggesting the presence of intraparenchymal gas. CT is the imaging procedure of choice in defining the extent of the emphysematous process and guiding management. Type I EPN is characterized by parenchymal destruction with either absence of fluid collection or presence of streaky or mottled gas radiating from the medulla to the cortex [4]; the absence of fluid collection implies a poor immune response and the mortality rate is high, at 66\%. Type II EPN typically has a confined, bubbly, intrarenal gas pattern-probably within abscesses associated with renal and perinephric fluid collection-and gas within the renal pelvis; the mortality rate in type II is $18 \%$ [5]. Although pneumaturia is absent unless the infection involves the collecting system, gas may extend beyond the site of inflammation to the sub capsular, perinephric and pararenal spaces extending into the scrotal sac and spermatic cord.

Conservative treatment alone, bilateral emphysematous pyelonephritis, type I EPN, and thrombocytopenia are associated with increased mortality [5]. Emphysematous pyelonephritis is a surgical emergency; most patients are septic, and fluid resuscitation and broad spectrum antimicrobial therapy are essential. If the kidney is functioning, medical therapy can be considered. Nephrectomy is mandatory for patients who do not improve after a few days of therapy; if the affected kidney is not functioning and not obstructed, nephrectomy should be performed because medical treatment alone is usually lethal. If a kidney is obstructed, catheter drainage must be instituted; if the patient's condition improves, nephrectomy may be deferred pending a complete urologic evaluation. In this respect, CT scan is diagnostic and is the method of choice for the diagnosis and follow-up. 
In our report, in our knowledge the first reported in literature with a rapid and acute worsening evolution, a fulminant EPN occurred within 24 hours from first clinical evaluation and needed urgent nephrectomy; despite a percutaneous nephrostomy was early placed the diabetes and immunosuppressive drugs worsened clinical condition and only CT scan allowed to perform the right diagnosis.

\section{Conclusion}

In the presence of patients at risk for urinary sepsis with worsening clinical conditions an early radiological (CT) and clinical evaluation should be performed to exclude the presence of EPB; moreover, in selected cases early nephrectomy should be mandatory to improve survival.

\section{Learning point}

- Emphysematous pyelonephritis (EPN) is an acute necrotizing parenchymal secondary to gas-forming uropathogens

- Computed Tomography (CT) is the imaging procedure of choice in defining the extent of the emphysematous process and guiding management

- In patients with urinary sepsis with worsening clinical conditions CT should be early performed to exclude the presence of EPB because, in selected cases, early nephrectomy should be mandatory.

\section{Conflicts of Interest}

The authors declare that they have no conflicts of interest. This research did not receive any specific grant from funding agencies in the public, commercial, or not-for-profit sectors.

\section{Ethical approval and consent to participate}

All procedures performed in this study involving human participants were in accordance with the ethical standards of the institutional and/or national research committee and with the 1964 Helsinki Declaration and its later amendments or comparable ethical standards.

\section{Informed consent}

Written informed consent was obtained from the patient for his anonymized information to be published in this article. 


\section{References}

1. Saxena D, Aggarwal L, Tudu SK, Shaji T (2013) Emphysematous Pyelonephritis - a Rare Surgical Emergency Presenting to the Physician: A Case Report and Literature Review. Indian J Surg 75: S272-S274.

2. Tsitouridis I, Michaelides M, Sidiropoulos D, Arvanity M (2010) Renal emphysema in diabetic patients: CT evaluation. Diagnostic and Interventional Radiology 16: 221-6.

3. Nabi T, Rafiq N, Rahman MHU, Rasool S, Wani NUD (2020) Comparative study of emphysematous pyelonephritis and pyelonephritis in type 2 diabetes: a single-centre experience. J Diabetes Metab Disord 19: 1273-82.

4. Huang JJ, Tseng CC (2000) Emphysematous pyelonephritis: clinicoradiological classification, management, prognosis, and pathogenesis. Arch Intern Med 160: 797-805.

5. Elawdy M, Osman Y, Abouelkheir RT, El-Halwagy S, Awad B, et al. (2019) Emphysematous pyelonephritis treatment strategies in correlation to the CT classification: have the current experience and prognosis changed? Int Urol Nephrol 51: 1709-13.

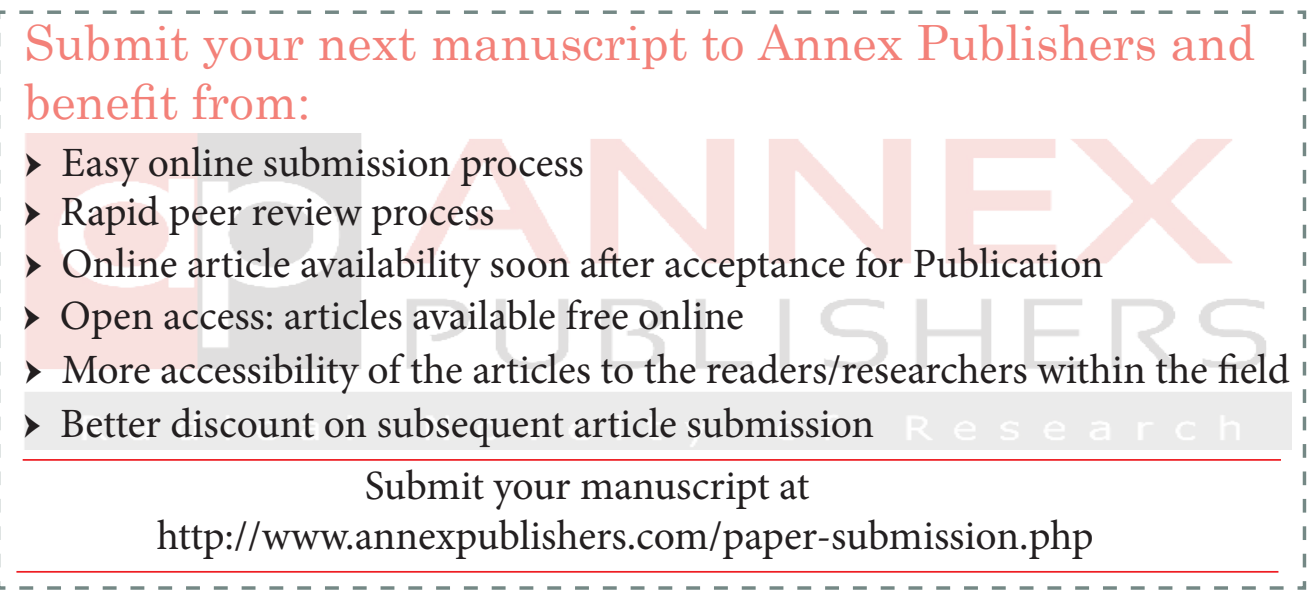

